\title{
A Semantic Mapping Method based on Ontology Structure Graph
}

\author{
Chengzhu Sun ${ }^{*}$ Weibin Chen,Bineng Zhong,Haibo Li \\ College of Computer Science and Technology, Huaqiao University, Xiamen 361021, China
}

\begin{abstract}
Keywords: Heterogeneity, Ontology structure graph,Model Interoperability, Semantic mapping Abstract. Efficient information sharing and exchanging are becoming increasingly important, but model heterogeneity hinder the communication and collaboration from different enterprises. To solve this problem, a semantic mapping method based on ontology structure graph is put forward in this paper. The requirement of model semantic interoperability among multiple enterprise model is analyzed, and model semantic interoperability is defined. Then, semantic clash between enterprise models is summarized and illustrated. Moreover, the formalization of semantic mapping rules is presented to support semantic mapping process from enterprise model to ontology. Besides, based on the description of ontology representation schema called ontology structure graph (OSG) and definition of semantic similarity function, semantic mapping process including model element choice, ontology elements selection, 3-level semantic matching based on OSG, semantic mapping rules generation, semantic mapping rules executing is illustrated in detail. Finally, the conclusions and future work are presented.
\end{abstract}

\section{Introduction}

As the model of each enterprise in the development process is mainly from the needs of their own enterprises, they use different modeling tools, different modeling methods, which produces a difficult problem in model data exchange among enterprise information system ${ }^{[1]}$, also because each enterprise model uses different model language description, which makes the enterprise model misunderstand the model, which seriously hindered the communication and collaboration from different enterprise information systems ${ }^{[2]}$. These are all the problems of interoperability of enterprise model, which is a challenging problem in the field of enterprise modeling.

The key to realize the interoperability is to achieve efficient information sharing and operation between the two information systems ${ }^{[3,4]}$. Ontology is the basis of semantic modeling, information integration and communication in the domain ${ }^{[5,6]}$. In order to realize enterprise interoperability, according to the local view, ontology provides a common understanding for the heterogeneous enterprise model $^{[7]}$. The ontology that supports the interaction of different enterprise models is a domain ontology, which is established by the corresponding domain experts. Here, domain ontology correctly represents the semantic information of the model elements in the process of enterprise interoperability ${ }^{[8]}$. Besides enterprise information system, semantic interoperability technique is widely used to solve information heterogeneity in some other system, such as mineral exploration $^{[9]}$, agricultural systems ${ }^{[10]}$,clinical decision support system ${ }^{[11]}$ etc.

The rest of this paper is organized as follows. In section 2, model semantic interoperability between enterprises is proposed and described. In section 3, semantic clash among enterprise models is illustrated in detail. In section 4, in order to support semantic mapping, semantic mapping rule is formalized. In section 5, based on the definition of ontology structure graph and semantic similarity function, semantic mapping process between enterprise models based on OSG is proposed and illustrated in detail. Finally, a summary of the conclusions and future work is presented.

\section{Model Semantic Interoperability among Enterprise Models}

The goal of model semantic interoperability is that although the initial development of the two enterprise models is not to collaborate, it is able to support the interaction of the two enterprises through the exchange of model data and services. Although they are likely to be the same model entities, as a result of the enterprise culture, database model, system structure, model description language and 
other important technical factors that hinder flexible collaboration, it may cause differences in the application of the model to understand the differences between the two enterprises.

Enterprise modeling is a process involving many departments, multiple stages, and repeated process. In the distributed enterprise environment, the heterogeneity among multiple enterprise models influences the knowledge sharing and collaboration among enterprises. Ontology is recognized as the main tool to solve the heterogeneity of knowledge. How to realize the understanding between different enterprises and the cooperation between different enterprise models is a key problem in the process of modeling and manufacturing process. The description of the semantic interoperability of the enterprise model is shown in Fig 1.

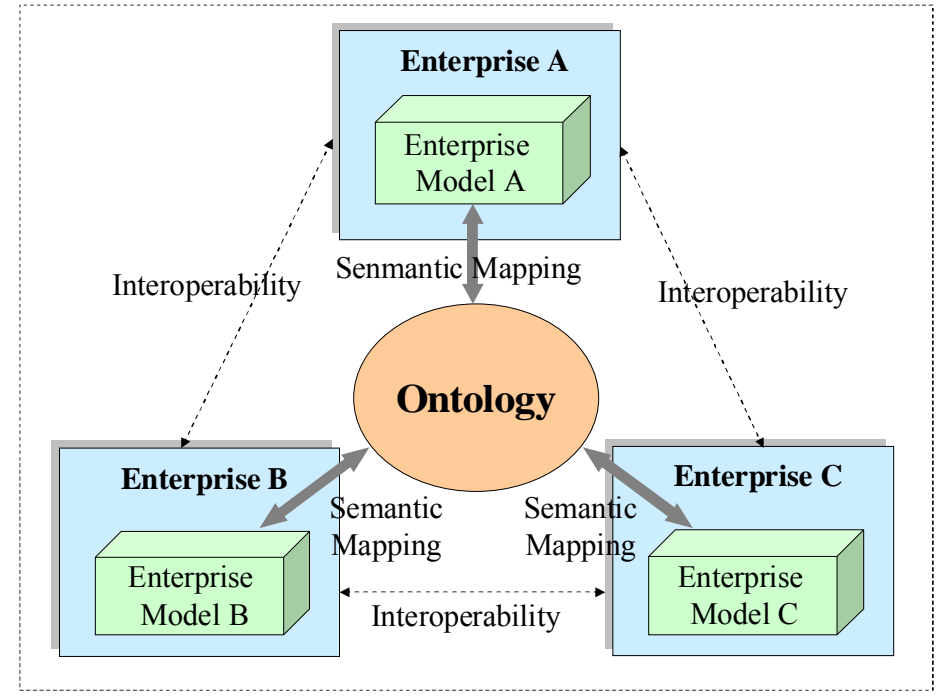

Fig 1. Model semantic interoperability between enterprise models

Given local model of $\mathrm{n}$ enterprises, $\mathrm{PM} \_\mathrm{Set}=\left\{\mathrm{EM}_{1}, \mathrm{EM}_{2}, \ldots, \mathrm{EM}_{\mathrm{n}}\right\}$, in order to achieve interoperability between enterprises, under the support of $\mathrm{EO}=\left\{\mathrm{OSG}_{1}, \mathrm{OSG}_{2}, \ldots \mathrm{OSG}_{\mathrm{m}}\right\}$ composed by $\mathrm{m}$ ontology fragments, for any two enterprise models of $\mathrm{EM}_{\mathrm{i}}$ and $E \mathrm{EM}_{\mathrm{j}}, \mathrm{EM}_{\mathrm{i}}, \mathrm{EM}_{\mathrm{j}} \in \mathrm{PM}$ _Set, here $\mathrm{i}$, $\mathrm{j}=1,2, \ldots, \mathrm{n}$, and $\mathrm{i} \neq \mathrm{j}$, semantic clash between the enterprise model can be analyzed through the semantic matching between enterprise model $\mathrm{EM}_{\mathrm{i}}$ and $\mathrm{EM}_{\mathrm{j}}$ to generate the semantic clash list $\mathrm{SCL}=\left\{<\mathrm{me}_{1}\right.$, $\left.\mathrm{me}_{2}>\mid \mathrm{me}_{1} \in \mathrm{EM}_{\mathrm{i}}, \mathrm{me}_{2} \in \mathrm{EM}_{\mathrm{j}}\right\}$. Finally with the help of to EO semantic mapping rules between two enterprise model $\mathrm{EM}_{\mathrm{i}}$ and $\mathrm{EM}_{\mathrm{j}}, \mathrm{MRS}=\left\{<\mathrm{me}_{1}, \mathrm{OE}, \mathrm{me}_{2}>\mid \mathrm{me}_{1} \in \mathrm{EM}_{\mathrm{i}}\right.$ and $\left.\mathrm{OE} \in \mathrm{EO}, \mathrm{me}_{2} \in \mathrm{EM}_{\mathrm{j}}\right\}$ can be established to support model semantic interoperability between multiple enterprises.

\section{Semantic Clash among Enterprise Models}

Among cooperative enterprises, the heterogeneity of the multiple enterprise models can produce the understanding difference of model semantic, which is the main reason that affects the interaction between cooperative enterprises. The semantic difference between the heterogeneous models is called semantic clash (SC). The semantic clash of the model is the characteristics of the different enterprise models, such as the name, attribute, type and precision etc. The goal of the semantic interoperability of enterprise model is to solve the semantic clash between model elements by semantic mapping.

Semantic clash occurs in two different levels: data level and schema level. Semantic clash of Data Level is the difference of the data field caused by multiple representation and interpretation of similar data. The semantic clash of Data Level includes data value clash, data representation clash, data unit clash, data precision clash. The semantic clash of schema level is the differences of the data logical structure or metadata (i.e., the schema) in the same application domain. This kind of semantic clash include naming clash, entity identity clash, schema, generalization clash, collection clash, and representation differences. In the semantic description of the enterprise model, besides the above concept level and schema level, it also considers relationship and constraints among model elements, which is called semantic clash of relation level. This semantic clash can be divided into three categories, such as inheritance associated clash, combination associated clash, and constraint associated clash. 
According to the above analysis, in order to explain the Interoperability requirements between the enterprise models, the semantic clash of the enterprise model are divided into three levels: data level conflict, schema level and link level, shown in Table1.

Table1. Semantic clashes between model elements

\begin{tabular}{|c|c|c|c|}
\hline \multicolumn{2}{|c|}{ Clash Type } & Clash Description & \multirow{2}{*}{$\frac{\text { Example }}{\text { Work-time of cylinder pad is not for } 10 \text { hours. }}$} \\
\hline $\begin{array}{l}\text { Data } \\
\text { Level }\end{array}$ & Value & $\begin{array}{l}\text { Inconsistency between element } \\
\text { values }\end{array}$ & \\
\hline \multirow[t]{3}{*}{ Clash } & Datatype & $\begin{array}{l}\text { Different data types to describe the } \\
\text { same entity attributes }\end{array}$ & String and long to show the phone number. \\
\hline & Unit & Different units of the attributes & Dollar and Pound for the same entity price \\
\hline & Precision & Accuracy of data representation & Integer and real number for work time \\
\hline \multirow[t]{5}{*}{$\begin{array}{l}\text { Schema } \\
\text { Level } \\
\text { Clash }\end{array}$} & Name & $\begin{array}{l}\text { Representing the same entity's model } \\
\text { elements and the concept of } \\
\text { Ontology }\end{array}$ & Material and raw materials for the resource \\
\hline & $\begin{array}{l}\text { Identificatio } \\
\mathrm{n}\end{array}$ & Different identity for same entity & Name and xingming for somebody's identity \\
\hline & Pattern & Different structure for same entity & The cylinder (time, cost, quantity) or other \\
\hline & Summary & A number of entities for an entity & Address and address of city and street \\
\hline & Set & A concept describe several entities & Length + width for cylinder head or other \\
\hline \multirow{3}{*}{$\begin{array}{l}\text { Relation } \\
\text { Level } \\
\text { Clash }\end{array}$} & Inheritation & Inheritation between model elements & The relation between car and moto-car \\
\hline & Combination & Inconsistency between two models & $\begin{array}{l}\text { Cylinder and cylinder head is not a } \\
\text { combination }\end{array}$ \\
\hline & Obligation & Model constraints between models. & Car and car manufacturing is no constraints \\
\hline
\end{tabular}

There are many reasons for the semantic clash of enterprise model, which may be due to the different knowledge expression of enterprise model elements, and it may be due to the different views of the modeling personnel and domain experts. An effective method to solve the semantic clash of the model is to establish the semantic mapping between clash model elements and solve the semantic clash among the multi enterprise models through semantic mapping rules.

\section{Semantic Mapping Rule}

Semantic Mapping Rule(SMR) is used to describe semantic relation between heterogeneous enterprise models. Model interoperability among enterprise models must be supported by an uniform model semantic expression. Semantic mapping rule is a valid solution to unify model semantic. A reference standard is given by reference ontology for the semantic of enterprise models. Semantic clash between model elements can be found by semantic match, then the mapping is established through model element-ontology and ontology-model element through mapping rules. Thus, semantic between two model elements is unified by solving semantic clash resovation. Semantic mapping rule is strictly defined according to specifical syntax as follows:

smr::=[source_model,target_model] valid_con sme

valid_con:: $=\overline{<}|=:|>$ :

sme::= sco(oe, cond) $\mid$ sco(oe, cond) \& sme $\mid$ sco(oe, cond) \# sme

here,

smr is semantic mapping rule: it is a expression to describe semantic clash between two heterogeneous models using domain ontology element. 
source_model is an element of source model: it is a model element in a enterprise model, maybe a conception, an attribute or a relation.

target_model is an element of target model: it is a model element in a enterprise model, maybe a conception, an attribute or a relation.

valid_con is a validation connector: it is used to connect two model elements and their semantic expression, showing the semantic of reference ontology expand $(<:)$, cover with $(=:)$ or not cover with $(>$ :) the semantic of two model elements;

sme is a semantic mapping expression: it is a recursive expression established according to reference ontology element and condition.

sco is a semantic clash symbol: it expresses semantic clash between model element and reference ontology element.

oe is an ontology element: it expresses a conception, an attribution or a relation of domain ontology. cond is a condition: it expresses the condition used by oe.

moreover, there are two logical connector that \& expresses AND and \# expresses OR, which is defined in se.

According to the formal description of the semantic mapping rules, the semantic expressions of the ontology elements and model elements that exist in the semantic clash can be defined, and the semantic representation of the ontology elements for solving the semantic clash of model elements is described.

\section{Semantic Mapping Method based on Ontology Structure Graph}

\section{Ontology Structure Graph}

To formalize knowledge in the enterprise, ontology is introduced to represent common schema. In our opinions, enterprise common schema is a composition of some ontology and rules among them. We conceptualize the composition as a graphic knowledge representation schema based on ontology, which is called Ontology Structure Graph $(\mathrm{OSG})^{[12]}$.

Definition1. An ontology structure graph is a graph $\mathrm{OSG}=<\mathrm{ONT}, \mathrm{L}, \mathrm{R}>$ such that,

ONT is a set of ontologies that represent objects describing general, domain-specific, task-specific knowledge of enterprise.

$\mathrm{L}$ is a set of links that connect two ontologies, $<\mathrm{ONT}, \mathrm{L}>$ is a directed graph.

$\mathrm{R}$ is a set of rules that define the constraints among ontologies of enterprise. Here, rules can be classified into three categories: algebraic rules, internal rules and external rules.

Definition2. An ontology representing knowledge of enterprise is a 5-tuple:

ONT:: $=\{$ Con, Attr, Val, Rel, Rul $\}$ such that,

Con is a set of conceptions according with terminology.

Attr is a set of attributes of conceptions.

Val is the value field of attributes.

Rel is a set of relations between two conceptions.

$\mathrm{Rul}$ is a set of rules among conceptions, attributes and values.

Definition 1 and definition 2 give a formalization of common schema for enterprise. OSG will be used to describe domain ontology as a reference between enterprise models.

\section{Semantic Similarity Function}

In the process of semantic mapping, model elements are compared with ontology elements to find the difference of their semantic. Semantic similarity function is defined to measure the difference between model element and ontology element quantitatively. For a model element A and an ontology element B, their similarity can be defined as follows:

$$
\operatorname{Sim}(\mathrm{A}, \mathrm{B})=\left\{\begin{array}{cl}
1 & \text { if } \mathrm{A}=\mathrm{B} \\
0.8 & \text { if } \mathrm{A} \text { is synonymous to } \mathrm{B} \\
0 & \text { otherwise }
\end{array}\right.
$$


Based on the similarity function above, the semantic similarity between partial model and ontology is defined as follows:

$$
\operatorname{SemSim}(\mathrm{M}, \mathrm{O})=\frac{2 \cdot \sum_{\mathrm{i}, \mathrm{j}} \operatorname{Sim}\left(\mathrm{E}_{\mathrm{M}}^{\mathrm{i}}, \mathrm{E}_{\mathrm{O}}^{\mathrm{j}}\right)}{\left|\mathrm{E}_{\mathrm{M}}\right|+\left|\mathrm{E}_{\mathrm{O}}\right|} \in[0,1]
$$

In equation (2), $\mathrm{M}$ is a part of model, and $\mathrm{O}$ is a part of ontology. $\mathrm{E}_{\mathrm{M}}^{\mathrm{i}}$ is one of the elements in the $\mathrm{M}$ and $\left|E_{M}\right|$ is the number of elements in the M. Semantic similarity function consists of equation (1) and equation (2), which is used to measure the difference between model fragment and ontology fragment.

\section{Semantic Mapping Process}

Model semantic mapping is the process of mapping the relationship between the two elements of the enterprise model, which is based on the semantic standard of domain ontology. In order to support the semantic interoperability of multiple enterprise models, a semantic mapping method based on OSG is proposed, as shown in Fig 2.

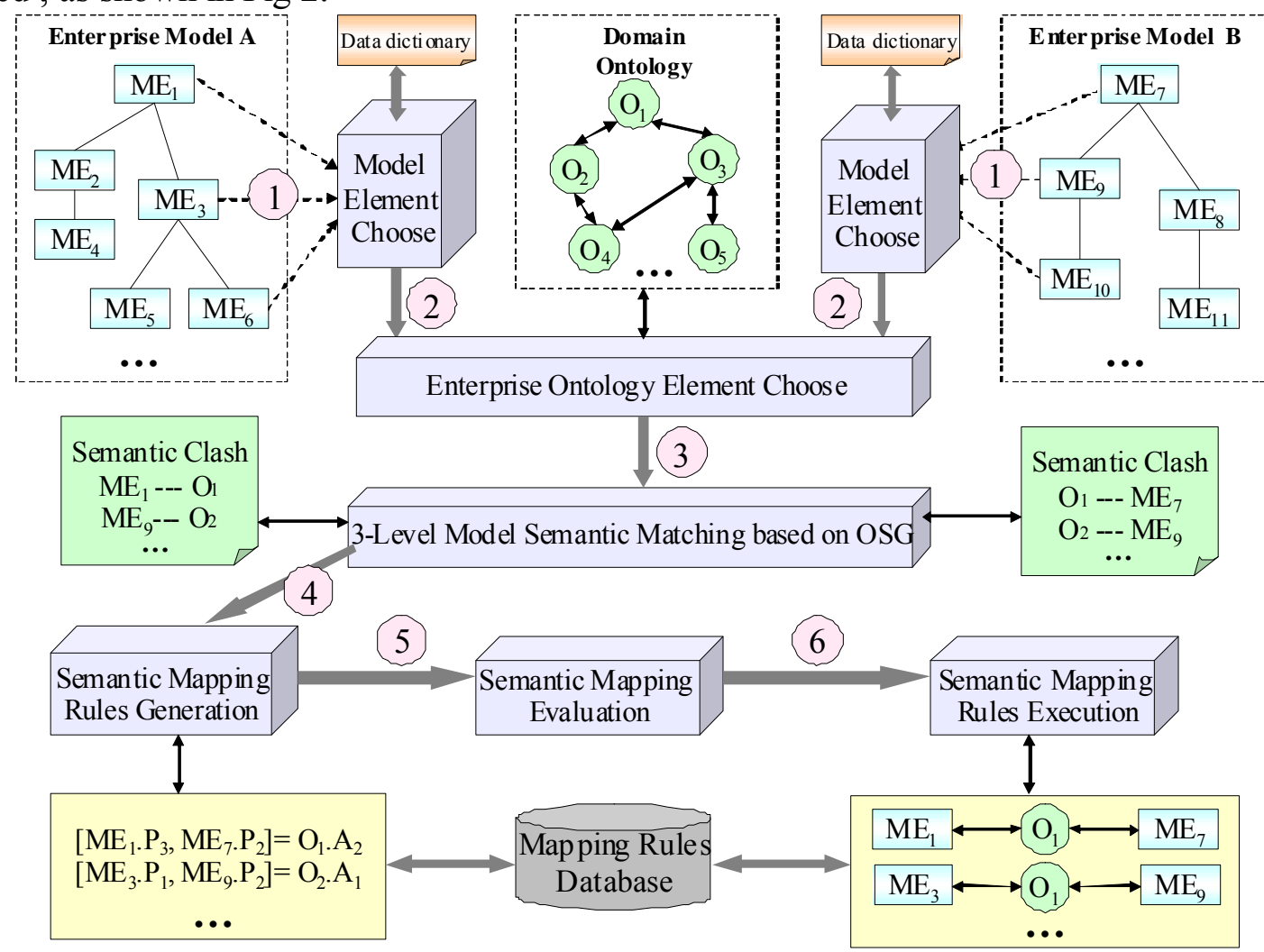

Fig 2. Semantic mapping process to enterprise model based on OSG

In the semantic mapping process (SMP) the data is from two enterprise model databases. According to the corresponding data dictionary of enterprise model the model elements are selected, and then, ontology elements in EO is searched according to the selected model elements to find semantic clash between model elements and domain ontology, and semantic mapping rules is generated and evaluated. Finally, the generated semantic mapping rules are stored in the mapping rule base, and the semantic mapping process of the two enterprise models is finished by running the mapping rules.

The semantic mapping process based on OSG consists of three stages: data preparation, semantic conflict discovery and semantic conflict resolution. The data preparation phase consists of two steps of SMP (1)and (2), and in this stage two enterprise model fragment specification is described. Semantic conflict discovery is refers to the step (3) of SMP, in this stage semantic conflict in the two model fragments is found in support of the domain ontology. Semantic conflict resolution phase is refers to the step (4) - (6) of SMP, in which interoperability between the two models is achieved through 
semantic mapping rules establishment, evaluation and execution. The six steps in the process of semantic mapping based on OSG are described as follows:

(1)Model element choose. In this step the model elements needed by the two enterprise interoperability is chosen to determine the model element attributes and model relation. OSG is used to represent the model data, and the model data is stored in the XML file.

(2) Ontology elements choose. In this step, the corresponding domain ontology fragments are determined based on the selected model elements to support a semantic reference for the semantic matching of two enterprise models.

(3) 3-Level semantic matching based on OSG. This is the core step of SMP. The semantic matching of the two enterprise models is carried out based on the similarity computation of OSG elements. Through the comparison of three levels including the data ,pattern and relation, a list of the semantic clash of the two models is generated.

(4) Semantic mapping rules generation. In this step, the list of semantic clash element of two model fragments is integrated according to the description of semantic mapping rules. Semantic mapping expression is generated to show the semantic clash in the two model fragments, and the relationship between the elements of the clash model is established.

(5) Semantic mapping evaluation. This step is to evaluate the validity of the generated semantic mapping rules, and to judge whether there is a lack of model semantics in the process of semantic mapping. The semantic mapping rules that pass the evaluation of the effectiveness can provide guidance for enterprise interoperability.

(6) Semantic mapping rules execution. The semantic relations among the two model elements is established by running the semantic mapping rules, and the semantic clash between the two model elements is solved. Thus, enterprise model interoperability are realized by the two levels mapping of model-ontology and ontology-model.

\section{Conclusions}

In this paper, a semantic mapping method based on ontology structure graph is proposed to support model interoperability between multiple enterprises. It is different from other semantic mapping methods, it extends the level of semantic clash, and adds relation level clash to semantic clash. In view of the characteristics of multi-enterprises, a new cross-domain ontology representation modelontology structure graph is used to describe the multi-domain ontology elements in the enterprise. Through semantic matching, semantic mapping, and the generation and execution of semantic mapping rules, the knowledge sharing and collaboration among distributed enterprises application information is realized. The semantic mapping method based on ontology structure graph presented in this paper provide a good solution for the model interoperability among the distributed enterprises.

Further research work includes: detailing of the semantic mapping process and semantic mapping algorithm; illustrating model interoperability to solve semantic clash using an application based on these theories.

\section{Acknowledgement}

Research works in this paper are partial supported by the National Natural Science Foundation of China (Grant No. 61572205), the Natural Science Foundation of Fujian Province, China (Grant No. 2015J01257, Grant No. 2012J05118), Science and Technology Platform Construction Project of Fujian Province, China(2012H2002), Quanzhou Science and Technology Plan Projects (No. 2015Z125) 


\section{References}

[1] Esma Yahia, Alexis Aubry, Hervé Panetto. Formal measures for semantic interoperability assessment in cooperative enterprise information systems. Computers in Industry, Volume 63, Issue 5, June 2012, pp443-457

[2] Virginie Fortineau, Thomas Paviot, Samir Lamouri. Improving the interoperability of industrial information systems with description logic-based models-The state of the art.Computers in Industry, Volume 64, Issue 4, May 2013, pp363-375

[3] Usman Wajid, Abdallah Namoun, César A. Marín, Nikolay Mehandjiev. Designing and evaluating a system of document recognition to support interoperability among collaborative enterprises.Computers in Industry, Volume 64, Issue 5, June 2013, pp 598-608

[4] Andrej Tibaut, Branko Kaučič, Danijel Rebolj. A standardised approach for sustainable interoperability between public transport passenger information systems.Computers in Industry, Volume 63, Issue 8, October 2012, pp788-798

[5] Bernardo Gonçalves, Giancarlo Guizzardi, José G. Pereira Filho. Using an ECG reference ontology for semantic interoperability of ECG data .Journal of Biomedical Informatics, Volume 44, Issue 1, February 2011, pp126-136

[6] Khouri Selma, Boukhari Ilyès, Bellatreche Ladjel, Sardet Eric, Jean Stéphane, Baron Michael. Ontology-based structured web data warehouses for sustainable interoperability: requirement modeling, design methodology and tool.Computers in Industry, Volume 63, Issue 8, October 2012, pp 799-812

[7] Ming Che Lee, Kun Hua Tsai, Tung Cheng Hsieh. A multi-strategy knowledge interoperability framework for heterogeneous learning objects . Expert Systems with Applications, Volume 38, Issue 5, May 2011, pp4945-4956

[8] Yves Ducq, David Chen, Guy Doumeingts. A contribution of system theory to sustainable enterprise interoperability science base.Computers in Industry, Volume 63, Issue 8, October 2012, pp 844-857

[9] Xiaogang Ma, Chonglong Wu, Emmanuel John M. Carranza, Ernst M. Schetselaar, Freek D. van der Meer, Gang Liu, Xinqing Wang, Xialin Zhang. Development of a controlled vocabulary for semantic interoperability of mineral exploration geodata for mining projects . Computers \& Geosciences, Volume 36, Issue 12, December 2010, pp1512-1522

[10] Edmund W. Schuster, Hyoung-Gon Lee, Reza Ehsani, Stuart J. Allen, J. Steven Rogers. Machine-to-machine communication for agricultural systems: An XML-based auxiliary language to enhance semantic interoperability. Computers and Electronics in Agriculture, Volume 78, Issue 2, September 2011, pp150-161

[11] Carlos Sáez, Adrián Bresó, Javier Vicente, Montserrat Robles, Juan Miguel García-Gómez. An HL7-CDA wrapper for facilitating semantic interoperability to rule-based Clinical Decision Support Systems .Computer Methods and Programs in Biomedicine, Volume 109, Issue 3, March 2013, pp239-249

[12] Chengzhu Sun, Xiaofei Xu, Shengchun Deng, Xiangyang Li. Knowledge Discovery for virtual enterprise based on Semantic Annotation. the 5 th International Conference on Fuzzy Systems and Knowledge Discovery (FSKD'08),Vol.5,2008,Jinan,Shandong, China, 18-20October 2008,pp546-551 